\title{
The impact of inulin as feed supplement on gut microbiota of honey bee (Apis mellifera)
}

\author{
Ákos JUHÁSZ ${ }^{1,2}$ - Alexandra VERESS ${ }^{1}$ - Orsolya ADAMCSIK ${ }^{1}$ - Szabolcs MOLNÁR ${ }^{2}$ - \\ Helga SZALONTAI ${ }^{2}$ \\ 1: Szent István University, Institute of Genetics, Microbiology and Biotechnology, Department of Microbiology \\ and Environmental Toxicology, H-2100 Gödöllö, Páter Károly street. 1.; E-mail: ajuhasz11@gmail.com \\ 2: Eszterházy Károly University, Food and Wine Research Centre, H-3300 Eger, Leányka street. 6.
}

Keywords: honey bee, prebiotics, gut microbiota

\section{Introduction}

Honey bee (Apis mellifera), as an important pollinator, contribute significantly to agricultural productions and the human food supply. Nowadays, bees are stressed by a number of biotic and abiotic factors (pathogens, pesticides, climate change, habitat loss, etc.) which affect health and productivity (Porrini et al., 2016; Alberoni et al., 2016). The European Union ban of antibiotics as feed additives has stimulated the search for natural alternatives. Animals have symbiotic microorganisms, which are important for the proper functioning of gastrointestinal tract (for nutrition and pathogen defence). This link between intestinal microbiota and health status, has encouraged researches to use beneficial gut microorganisms and prebiotics, to improve the health of animals (and humans). Probiotics (mainly lactic acid bacteria) and prebiotics (like inulin) help to protect the intestinal microbial. There are commercial probiotics and/or prebiotics containing diet supplements in beekeeping management too, but most of them have been developed for humans and the differences between the gut microbiota of honey bees and vertebrates may cause an opposite effect (Ptaszyńska et al., 2016). The intestinal system of honey bee contains relatively few bacteria in number and variety. Eight major bacterial groups concentrated in the hindgut: two Alpha-, one Beta- and two Gammaproteobacteria, two closely related Lactobacillus group (Firm 4, 5), and one Bifidobacterium (Moran, 2015). Some of them are found in all honey bees (Lactobacilli, Beta- and Gammaproteobacteria), but the other groups of bacteria may be completely absent from some animals/individuals (Moran et al., 2012).

Our aim was to investigate the effect of a commercial inulin, as a nutritional supplement, to the composition of gut microbiota of honey bee.

\section{Materials and methods}

The control group was fed with one litre sucrose syrup ( $2 \mathrm{~kg}$ sugar in 3 litre water) every second day. Treated groups got the same feed but 30 or $60 \mathrm{~g} \mathrm{l}^{-1}$ inulin was added to the syrup. The treatment was performed with three colonies in both cases and the samples (approximately 20-20 worker bees) were collected before the treatment and 2, 4 and 6 weeks later, depending on the duration of the treatment. The intestinal tracts of five bees were isolated, homogenised and diluted in $1 \%$ tripton containing saline. Traditional microbiological methods were used to determine the amount of living microbes. We used six different media (Nutrient agar, Compass Enterococcus agar, De Man, Rogosa and Sharpe agar, Eosin Methylene Blue agar, Chromobio Coliform agar and Bifidobacterium medium) to investigate total aerob bacteria, Lactobacillus, Enterococcus, Bifidobacterium, Eschericchia coli and coliform bacteria. 


\section{Results and discussion}

We have compared the repeatability of sample processing in case of one and five animals. The complete intestinal system was isolated, homogenised and the colony numbers of bacteria was normalised to one gut. In case of individual isolates, the deviation is very high, especially in case of coliform bacteria $\left(2.0 \times 10^{2}-1.3 \times 10^{7}\right)$ and Enterococcus genus. We found that the difference between parallel samples containing five homogenized gut per sample, with the exception of E. coli and coliform bacteria, was smaller than for single intestinal samples. Our results show, that the pooling of five intestines gives a more reproducible result. Apart from the differences in the individual animals, the individual honey bee colonies also differed significantly, making the results obtained for several hives incomparable. The literature also confirms that there are very large differences between the intestinal microbes of individual animals in case of honey bee (Moran et al., 2012). Based on our results, two hives were investigated and the exact colony count were not compared, only the tendency of changes due to different treatments was calculated. The bees were treated with sugar syrup and then with increasing inulin concentration, but the effect of the inulin was not detectable. We found that the amount of certain bacteria (total aerob bacteria and Lactobacillus) increased significantly (ten to hundredfold) as a result of the sugar syrup treatment. We will carry out further tests to prove our results in spring 2019.

\section{Conclusions}

We have developed a method that can be used to test the changes of gut microbiota in honey bee. The prebiotic effect of inulin was not detected by the presently used technique, but we will test more prebiotics in the future.

\section{Acknowledgement}

This research was supported by the grant 2017-1.3.1-VKE-2017-00001 and EFOP-3.6.116-2016-00001.

\section{References}

Alberoni, D., Gaggia, F., Baffoni, L., Di Gioia, D. (2016): Beneficial microorganisms for honey bees: problems and progresses. Applied Microbiology and Biotechnology. 100: 9469-9482. DOI 10.1007/s00253-016-78704

Porrini, C., Mutinelli, F., Bortolotti, L., Granato, A., Laurenson, L., Roberts, K., Gallina, A., Silvester, N., Medrzycki, P., Renzi, T., Sgolastra, F., Lodesani, M. (2016): The status of honey bee health in Italy: results from the nationwide bee monitoring network. PLoS One 11: 5. e0155411. doi:10.1371/journal.pone.0155411

Moran, N.A. (2015): Genomics of the honey bee microbiome. Current Opinion in Insect Science 10: $22-28$. https://doi.org/10.1016/j.cois.2015.04.003

Moran, N.A., Hansen, A.K., Powell, J.E., Sabree, Z.L. (2012): Distinctive gut microbiota of honey bees assessed using deep sampling from individual worker bees. PLoS ONE 7: 4. e36393. https://doi.org/10.1371/journal. pone. 0036393

Ptaszyńska, A.A., Borsuk, G., Zdybicka-Barabas, A., Cytryńska, M., Małek, W. (2016): Are commercial probiotics and prebiotics effective in the treatment and prevention of honeybee nosemosis C? Parasitology Research 115: 397-406. doi:10.1007/s00436-015-4761-z 